Pacific Journal of Mathematics

ON THE VECTOR FIELDS ON AN ALGEBRAIC 


\title{
ON THE VECTOR FIELDS ON \\ AN ALGEBRAIC HOMOGENEOUS SPACE
}

\author{
YoshifUMi KaTO
}

\begin{abstract}
We construct a holomorphic vector field $V$ with isolated zeros on an algebraic homogeneous space $X=G / P$ and show that the Koszul complex defined by $V$ gives much information concerning the cohomology groups of $X$. Our results give useful examples to the studies of $\mathrm{J}$. B. Carrell and D. Lieberman.
\end{abstract}

1. Koszul complex. Let $X$ be a compact Kähler manifold of dimension $n$. We assume the manifold $X$ admits a holomorphic vector field $V$ whose zero set $Z$ is simple isolated and nonempty. The following complex of sheaves is said to be the Koszul complex defined by $V$ :

$$
0 \rightarrow \Omega^{n} \stackrel{\partial}{\rightarrow} \Omega^{n-1} \stackrel{\partial}{\rightarrow} \cdots \rightarrow \Omega^{1} \stackrel{\partial}{\rightarrow} \Omega^{0}=\theta_{X} \rightarrow 0
$$

where the differential $\partial$ is the contraction map $i(V)$. The structure sheaf of $Z$ is $\theta_{Z}=\theta_{X} / i(V) \Omega^{1}$. To make the differentials of degree +1 , we substitute $K^{p}=\Omega^{-p}$ :

$$
0 \rightarrow K^{-n} \stackrel{\partial}{\rightarrow} K^{-n+1} \stackrel{\partial}{\rightarrow} \ldots \stackrel{\partial}{\rightarrow} K^{0} \rightarrow 0 .
$$

For any locally free sheaf $\mathscr{F}$, we denote by $K(\mathscr{F})$ the complex obtained by tensoring $\mathscr{F}$ with (1.2) over $\theta_{X}$. Let $\mathcal{H}^{*}(\mathscr{F})$ be the cohomology sheaves of the complex $K(\mathscr{F})$. Then, from the assumptions, it follows that $\mathcal{H}^{q}(\mathscr{F})=0$ for $-n \leq q<0$ and $\mathcal{H}^{0}(\mathscr{F})=\mathscr{F} \otimes \mathcal{O}_{Z}$, whose support is contained in $Z$. We abbreviate $\mathscr{F}_{Z}=\mathscr{F} \otimes \mathcal{O}_{Z}$. The hypercohomology $\mathbf{H}^{*}(X, K(\mathscr{F}))$ can be calculated by using the double Čech complex $\check{C}^{*}(\mathcal{Q}, K(\mathscr{F}))$ in the usual manner. See [3]. Corresponding to the natural two filtrations in $\check{C}^{*}(\mathcal{Q}, K(\mathscr{F}))$, we get the following spectral sequences which converge to $\mathbf{H}^{p+q}(X, K(\mathscr{F}))$ :

$$
\begin{aligned}
{ }^{\prime} E_{1}^{p, q} & =H^{q}\left(X, K^{p}(\mathscr{F})\right), \\
{ }^{\prime} E_{2}^{p, q} & =H^{p}\left(X, \mathcal{H}^{q}(\mathscr{F})\right) .
\end{aligned}
$$

From the above remark, it follows that $\mathbf{H}^{r}(X, K(\mathscr{F}))=0$ for $r \neq 0$ and $\mathbf{H}^{0}(X, K(\mathscr{F}))=H^{0}\left(Z, \mathscr{F}_{Z}\right)$. Note that the space $H^{0}\left(Z, \vartheta_{Z}\right)$, i.e., in case $\mathcal{F}=\theta_{X}$, can be interpreted as the ring of complex-valued functions on $Z$. 
Let $\mathscr{F}_{1}, \mathscr{F}_{2}$ and $\widetilde{F}_{3}$ be locally free sheaves and $\phi: \mathscr{F}_{1} \times \mathscr{F}_{2} \rightarrow \mathscr{F}_{3}$ a bilinear map. Then by using the exterior product in $K^{*}$, we obtain a bilinear map

$$
\phi: \mathbf{H}^{p}\left(X, K\left(\mathscr{F}_{1}\right)\right) \times \mathbf{H}^{q}\left(X, K\left(\mathscr{F}_{2}\right)\right) \rightarrow \mathbf{H}^{p+q}\left(X, K\left(\mathscr{F}_{3}\right)\right) .
$$

Further if we denote by $F \mathbf{H}^{p}\left(K\left(\mathscr{F}_{i}\right)\right)$ the filtration on $\mathbf{H}^{p}\left(X, K\left(\mathscr{F}_{i}\right)\right)$ induced from the ' $E_{1}$-terms (1.3), then the map keeps the filtrations

$$
\phi: F_{r} \mathbf{H}^{p}\left(K\left(\mathscr{F}_{1}\right)\right) \times F_{s} \mathbf{H}^{q}\left(K\left(\mathscr{F}_{2}\right)\right) \rightarrow F_{r+s} \mathbf{H}^{p+q}\left(K\left(\mathscr{F}_{3}\right)\right)
$$

for $p, q, r, s \in \mathbf{Z}$. In particular if we take $\mathscr{F}_{i}=\vartheta_{X}, 1 \leq i \leq 3$, and $\phi$ : $\theta_{X} \times \theta_{X} \rightarrow \theta_{X}$ the multiplication, we can introduce a natural ring structure in $\mathbf{H}^{0}(X, K)$ which is compatible with the wedge product pairing of the groups ' $E_{1}^{-p, q}=H^{q}\left(X, \Omega^{p}\right)$. Further we have the following known results. See [2], [3].

LEMMA 1. Suppose the manifold $X$ and the vector field $V$ are as above. Then

(1) In case $\mathscr{F}=\mathcal{O}_{X}$, all the differentials of (1.3) vanish.

(2) Therefore comparing (1.3) and (1.4), we have

$$
H^{p}\left(X, \Omega^{q}\right)=0 \text { for } p \neq q .
$$

(3) The space $H^{0}\left(Z, \theta_{Z}\right)$ has the canonical filtration induced from the filtered hypercohomology ring $\mathbf{H}^{0}(X, K)$ such that:

$$
\begin{gathered}
H^{0}\left(Z, \Theta_{Z}\right)=F_{-n} \supseteq F_{-n+1} \supseteq \cdots \supseteq F_{0} \supseteq\{0\}, \\
F_{p} \cdot F_{q} \subseteq F_{p+q}, \\
F_{-p} / F_{-p+1} \cong H^{p}\left(X, \Omega^{p}\right), \\
H^{*}(X, \mathbf{C}) \cong \operatorname{gr} H^{0}\left(Z, \Theta_{Z}\right)=\bigoplus_{p=0}^{n} F_{-p} / F_{-p+1} .
\end{gathered}
$$

2. $V$-equivariant vector bundles. The following definition and results are in [3].

Definition. We say that a vector bundle $\mathcal{E}$ on $X$ is $V$-equivariant if the derivation $V: \vartheta_{X} \rightarrow \vartheta_{X}$ can be lifted to $\mathscr{E}$, i.e., there exists a $\mathbf{C}$-linear $\operatorname{map} \tilde{V}: \mathcal{E} \rightarrow \mathcal{E}$ such that

$$
\tilde{V}(f \cdot s)=V(f) \cdot s+f \cdot \tilde{V}(s)
$$

where $f$ is a local section of $\theta_{X}$ and $s$ that of $\varepsilon$. 
Let $\left\{f_{i j}\right\}$ be a set of transition matrices of $\mathcal{E}$. Then the set $\left\{d f_{i j} \cdot f_{i j}^{-1}\right\}$ defines the Atiyah-Chern class $c(\mathcal{E})$ of $\mathcal{E}$ in $H^{1}\left(X, \operatorname{Hom}(\mathcal{E}, \mathcal{E}) \otimes \Omega^{1}\right)$. And the class $i(V) c(\mathcal{E})$ in $H^{1}(X, \operatorname{Hom}(\mathcal{E}, \mathcal{E}))$ is the obstruction for $\mathscr{E}$ to be $V$-equivariant. See [3]. If we put $\mathscr{F}=\operatorname{Hom}(\mathcal{E}, \mathcal{E})$, the cohomology groups $H^{1}\left(X, \operatorname{Hom}(\mathcal{E}, \mathcal{E}) \otimes \Omega^{1}\right)$ and $H^{1}(X, \operatorname{Hom}(\mathcal{E}, \mathcal{E}))$ can be interpreted as the ' $E_{1}^{-1,1}$ and ' $E_{1}^{0,1}$-terms, respectively, of the spectral sequence (1.3). Therefore each $V$-equivariant vector bundle $\delta$ defines the hypercohomology class $\tilde{c}(\mathcal{E})$ lying in $F_{-1} \mathbf{H}^{0}(X, K(\operatorname{Hom}(\mathcal{E}, \mathcal{E})))$. Here the class $\tilde{c}(\mathcal{E})$ is well defined only up to $F_{0} \mathbf{H}^{0}(X, K(\operatorname{Hom}(\mathcal{E}, \mathcal{E})))$ and is called the hyper-Chern class of $\mathcal{E}$. We denote by $\sigma_{d}: \operatorname{Hom}(\mathcal{E}, \mathcal{E}) \rightarrow \Theta_{X}, 0 \leq d \leq r=$ rank $\mathcal{E}$, the vector bundle homomorphisms defined by the rule:

$$
\operatorname{det}(t I+\mathscr{Q})=\sum_{d=0}^{r} \sigma_{d}(\mathscr{Q}) t^{r-d}, \quad \mathscr{Q} \in \operatorname{Hom}(\mathcal{E}, \mathcal{E})
$$

The mapping $\sigma_{d}$ is usually called the $d$ th elementary function and is a polynomial map of degree $d$. We denote by $e_{d}: F_{-d} \mathbf{H}^{0}(X, K) \cong F_{-d} \rightarrow$ $H^{d}\left(X, \Omega^{d}\right)$ the mapping which induces the canonical isomorphism $F_{-d} / F_{-d+1} \cong H^{d}\left(X, \Omega^{d}\right)$.

Lemma 2. The map $\sigma_{d}$ determines the classes $\sigma_{d}(c(\mathcal{E}))$ and $\sigma_{d}(\tilde{c}(\mathcal{E}))$ which belong to $H^{d}\left(X, \Omega^{d}\right)$ and $F_{-d} \mathbf{H}^{0}(X, K)$, respectively. We have:

(1) $(-1)^{d} \sigma_{d}(c(\mathcal{E}))$ is the dth Chern class of $\mathcal{E}$ and coincides with $(-1)^{d} e_{d}\left(\sigma_{d}(\tilde{c}(\tilde{E}))\right)$.

(2) Let $\tilde{V}_{Z} \in H^{0}\left(Z, \operatorname{Hom}(\mathcal{E}, \mathcal{E})_{Z}\right) \cong \mathbf{H}^{0}(X, K(\operatorname{Hom}(\mathcal{E}, \mathcal{E})))$ denote the restriction of $\tilde{V}$ to $Z$. Then $(-1)^{d} \sigma_{d}\left(\tilde{V}_{Z}\right)$ belongs to $H^{0}\left(Z, \theta_{Z}\right)$ and is equal to $(-1)^{d} \sigma_{d}(\tilde{c}(\mathscr{E}))$.

3. Semisimple Lie algebras. Let $g$ be a complex semisimple Lie algebra. We choose a compact form $t$ and define a $*$-operation on $g$ with respect to $t$. Let $\mathfrak{b}$ be a Borel subalgebra of $\mathfrak{g}$. If we put $\mathfrak{h}=\mathfrak{b} \cap \mathfrak{b}^{*}$ then $\mathfrak{h}$ becomes a Cartan subalgebra of $\mathfrak{g}$. Let $\Delta \subset \mathfrak{h}_{\mathfrak{R}}^{*}$ be the root system of $\mathfrak{h}$ in $\mathrm{g}$. The set $\Delta$ is divided into two classes, the positive roots $\Delta_{+}$and negative roots $\Delta_{-}$with respect to $\mathfrak{b}$. We denote by $\Pi$ the set of simple roots corresponding to $\Delta_{+}$. Then any root $\phi \in \Delta$ can be written as $\phi=$ $\sum_{\alpha \in \Pi} n_{\alpha}(\phi) \alpha$ where $n_{\alpha}(\phi)$ are nonnegative or nonpositive integers according to $\phi \in \Delta_{+}$or $\Delta_{-}$. The algebra $g$ has the root space decomposition

$$
\mathfrak{g}=\mathfrak{h}+\sum_{\alpha \in \Delta_{+}} \mathfrak{g}_{\alpha}+\sum_{\beta \in \Delta_{-}} \mathfrak{g}_{\beta}
$$


where

$$
\mathfrak{b}=\mathfrak{h}+\sum_{\alpha \in \Delta_{+}} \mathfrak{g}_{\alpha} .
$$

For any $\alpha \in \Delta, \operatorname{dim} \mathfrak{g}_{\alpha}=1$, and from the definition of $\mathfrak{g}_{\alpha}$ it follows that

$$
\operatorname{ad}(H)(X)=[H, X]=\alpha(H) X, \quad X \in \mathfrak{g}_{\alpha}, H \in \mathfrak{h} .
$$

Let $\mathfrak{p}$ be a parabolic Lie subalgebra of $\mathfrak{g}$ which contains $\mathfrak{b}$. Then there exists a decomposition of $\mathfrak{g}$ corresponding to $\mathfrak{p}$.

LEMma 3. Let $\mathfrak{g}, \mathfrak{p}$ be as above. We put $\mathfrak{n}=\{Z \in \mathfrak{g} \mid(Z, Y)=0$ for all $Y \in \mathfrak{p}\}$ where (,) is the Killing form of $\mathrm{g}$. Then $\mathfrak{n}$ is the maximal nilpotent ideal of $p$ and also the set of all nilpotent elements in the radical of $p$. Further if we define $\mathfrak{g}_{1}=\mathfrak{p} \cap \mathfrak{p}^{*}$, then we have the decomposition

$$
\mathrm{g}=\mathfrak{n}^{*}+\mathrm{g}_{1}+\mathfrak{n}, \quad \mathfrak{p}=\mathfrak{g}_{1}+\mathfrak{n} .
$$

Moreover $\mathrm{g}_{1}$ lies in the normalizers of both $\mathfrak{n}$ and $\mathfrak{n}^{*}$.

For any subspace a which is invariant by the adjoint action of $\mathfrak{h}$, we define the set $\Delta(\mathfrak{a}) \subseteq \Delta$ as follows:

$$
\Delta(\mathfrak{a})=\left\{0 \neq \alpha \in \mathfrak{h}_{\Re}^{*} \mid[H, X]=\alpha(H) X \text { for some } 0 \neq X \in \mathfrak{a}\right.
$$

and any $H \in \mathfrak{h}$ \}.

The subalgebras $\mathfrak{g}_{1}, \mathfrak{n}$ and $\mathfrak{n}^{*}$ are invariant and we have:

$$
\begin{gathered}
\Delta\left(\mathfrak{g}_{1}\right)=\left\{\phi \in \Delta \mid n_{\alpha}(\phi)=0 \text { for all } \alpha \in \Pi \cap \Delta(\mathfrak{p})\right\}, \\
\Delta(\mathfrak{n})=\left\{\phi \in \Delta_{+} \mid n_{\alpha}(\phi)>0 \text { for all } \alpha \in \Pi \cap \Delta(\mathfrak{p})\right\}, \\
\Delta\left(\mathfrak{n}^{*}\right)=-\Delta(\mathfrak{n}) .
\end{gathered}
$$

Let $G$ be a simply-connected complex semisimple Lie group whose Lie algebra is $g$. Let $B, T$ and $P$ be the Borel subgroup of $G$ with Lie algebra $\mathfrak{b}$, the Cartan subgroup with Lie algebra $\mathfrak{h}$, and the parabolic subgroup with Lie algebra $\mathfrak{p}$, respectively. The homogeneous space $X=$ $G / P$ becomes compact. Further, the space $X$ can be embedded into a certain projective space by using the representation theory of $G$. Hence we call the space $X$ an algebraic homogeneous space. Let $G_{1}, N$ and $N^{*}$ be the Lie subgroups of $G$ corresponding to $\mathfrak{g}_{1}, \mathfrak{n}$ and $\mathfrak{n}^{*}$, respectively. Then the group $P$ is the semidirect product of $G_{1}$ and $N$, and, further, $P \cap N^{*}=$ $\{I\}$. See [7].

Let $N(T)$ be the normalizer of $T$ in $G$. We call the group $W=N(T) / T$ the Weyl group of $G$ with respect to $T$. We put $W_{1}=N(T) \cap P / T \subset W$ 
and $W^{1}=W / W_{1}$. The group $N(T)$ acts on $T, \mathfrak{h}$ and $\Delta$ as follows:

$$
\begin{aligned}
& \mathfrak{w} \cdot \exp H \cdot \mathfrak{w}^{-1}=\exp (\operatorname{Ad}(\mathfrak{w}) H), \\
& \left(\operatorname{Ad}(\mathfrak{w})^{*} \alpha\right)(H)=\alpha\left(\operatorname{Ad}(\mathfrak{w})^{-1} H\right),
\end{aligned}
$$

for $\mathfrak{w} \in N(T), H \in \mathfrak{h}, \alpha \in \Delta$. But if $\mathfrak{w} \in T$, the actions of $\mathfrak{w}$ are all trivial. Hence we can regard as the group $W$ acts on $T, \mathfrak{h}$ and $\Delta$. For simplicity, we use the same letter $\mathfrak{w}$ for $\mathfrak{w}, \operatorname{Ar}(\mathfrak{w})$ and $\operatorname{Ad}(\mathfrak{w})^{*}$.

4. Main results. We first prove the following proposition.

Proposition 1. If we act the maximal torus $T$ on $X=G / P$ then the set $W^{1}=W / W_{1}=N(T) / N(T) \cap P$ is naturally realized as the set of all $T$ fixed points in $X$.

Proof. An element $\bar{g} \in X$ is fixed by the action of $T$ if and only if $g^{-1} \mathrm{Tg} \subset P$ where $g$ is a representative of $\bar{g}$ in $G$. Since the group $g^{-1} T g$ is also a maximal torus of $G$ contained in $P$, there exists $p \in P$ such that $g^{-1} T g=p T g^{-1}$. This means $g p \in N(T)$. Hence $\bar{g}$ defines a coset $\widetilde{g p}$ in $W^{1}$. If two fixed points $\bar{g}$ and $\bar{g}^{\prime}$ define the same coset in $W^{1}$ then $g p=g^{\prime} p^{\prime} p^{\prime \prime}$ for some $p, p^{\prime} \in P$ and $p^{\prime \prime} \in N(T) \cap P$. So $\bar{g}=\bar{g}^{\prime}$ in $X$. If we take an element $\mathfrak{w} \in N(T)$ then the coset corresponding to $\overline{\mathfrak{w}}$ is $\tilde{\mathfrak{w}} \in W^{1}$. Hence the mapping is onto.

Let us consider the following diagram:

\begin{tabular}{|c|c|c|c|c|c|c|}
\hline & & $G$ & & $G / P$ & & $G / P$ \\
\hline & & $\cup$ & & $U$ & & $U$ \\
\hline$n^{*}$ & $\stackrel{\phi}{\rightarrow}$ & $N^{*}$ & $\stackrel{\psi}{\rightarrow}$ & $\bar{N}^{*}$ & $\stackrel{\mathfrak{m}}{\rightarrow}$ & $\mathfrak{m} \bar{N}^{*}$ \\
\hline$\Psi$ & & $\Psi$ & & $\Psi$ & & $\Psi$ \\
\hline 7 & $\rightarrow$ & $\exp Z$ & $\rightarrow$ & $\overline{\exp Z}$ & $\rightarrow$ & $\exp Z$ \\
\hline
\end{tabular}

We write an element $Z$ of $\mathfrak{n}^{*}$ as $Z=\sum_{\alpha \in \Delta\left(\mathfrak{n}^{*}\right)} z_{\alpha} X_{\alpha}$ with respect to the basis $X_{\alpha} \in \mathrm{g}_{\alpha}, \alpha \in \Delta\left(\mathrm{n}^{*}\right)$, of $\mathrm{n}^{*}$. Since the Lie algebra $\mathrm{n}^{*}$ is nilpotent, we have $\log (\exp Z)=Z$ and hence the map $\phi$ is one-to-one and onto. Since $N^{*} \cap P=\{I\}$, the mapping $\psi$ is also one-to-one. The left multiplication of $\mathfrak{w}$ is clearly one-to-one. Hence we can take the pair $\left(\mathfrak{w} \bar{N}^{*}, \phi^{-1} \circ \psi^{-1} \circ \mathfrak{w}^{-1}\right)$ as a coordinate neighborhood near $\mathfrak{w} \in W^{1}$ and then the functions $\left\{z_{\alpha}\left(\mathfrak{w} \bar{n}^{*}\right)\right\}_{\alpha \in \Delta\left(\mathrm{n}^{*}\right)}$ become the local coordinates. 
THEOREM 1. The quotient set $W^{1}=W / W_{1}$ can be canonically embedded into $X=G / P$ as the set of all T-fixed points, and the pair $\left(\mathfrak{m} \bar{N}^{*}, \phi^{-1} \circ \psi^{-1} \circ \mathfrak{m}^{-1}\right)$ is a coordinate neighborhood near $\mathfrak{m} \in W^{1}$. The sets $\mathfrak{w} \bar{N}^{*}, \mathfrak{w} \in W^{1}$, are all $T$ invariant Zariski open sets. In fact if we multiply $\exp H \in T$ on $\mathfrak{m} \bar{N}^{*}$, the local coordinate $\left\{z_{\alpha}\left(\mathfrak{w} \bar{n}^{*}\right)\right\}_{\alpha \in \Delta\left(\mathfrak{n}^{*}\right)}$ changes to $\left\{e^{(\mathrm{m} \alpha)(H)} \cdot z_{\alpha}\left(\mathrm{m} \bar{n}^{*}\right)\right\}_{\alpha \in \Delta\left(\mathrm{n}^{*}\right)}$. Further, the space $X$ is covered with the family of the open sets $\mathfrak{w} \bar{N}^{*}$, i. e., $X=\bigcup_{\mathfrak{w} \in W^{1}} \mathfrak{w} \bar{N}^{*}$.

Proof. The first sentence has been proved. Let $\mathrm{m}_{0}$ be the element of $W$ whose length is maximal among all. Then since $\mathfrak{m}_{0}^{-1} N \mathfrak{w}_{0}=N^{*}, \mathfrak{w}_{0} \bar{N}^{*}=$ $N \mathfrak{w}_{0} P / P$. Namely the set $\mathfrak{w}_{0} \bar{N}^{*}$ is the Bruhat cell of maximal dimension and is a Zariski open set. So $\mathfrak{w} \bar{N}^{*}=\mathfrak{w m}_{0}^{-1} \mathfrak{w}_{0} \bar{N}^{*}, \mathfrak{m} \in W^{1}$, are all Zariski open sets. Since, for $\exp Z \in N^{*}$,

$$
\begin{aligned}
\exp H \cdot \mathfrak{w} \exp Z \cdot P & =\mathfrak{w} \mathfrak{w}^{-1} \exp H \mathfrak{w} \cdot \exp Z \cdot \mathfrak{w}^{-1} \exp (-H) \mathfrak{w} \cdot P \\
& =\mathfrak{w} \cdot \exp \left(\mathfrak{w}^{-1}(H)\right) \cdot \exp Z \cdot \exp \left(-\mathfrak{w}^{-1}(H)\right) \cdot P \\
& =\mathfrak{w} \cdot \exp \left(\operatorname{Ad}\left(\exp \left(\mathfrak{w}^{-1}(H)\right)\right) Z\right) \cdot P \\
& =\mathfrak{w} \cdot \exp \left(\operatorname{Exp}\left(\operatorname{ad}\left(\mathfrak{w}^{-1}(H)\right)\right) Z\right) \cdot P
\end{aligned}
$$

and

$$
\operatorname{Exp}\left(\operatorname{ad}\left(\mathfrak{w}^{-1}(H)\right)\right) \cdot Z \in \mathfrak{n}^{*}
$$

then

$$
\left(\phi^{-1} \circ \psi^{-1} \circ \mathfrak{m}^{-1}\right)(\exp H \cdot \mathfrak{m} \overline{\exp Z})=\operatorname{Exp}\left(\operatorname{ad}\left(\mathfrak{m}^{-1}(H)\right)\right) \cdot Z .
$$

If we write $Z=\sum_{\alpha \in \Delta\left(\mathfrak{n}^{*}\right)} z_{\alpha} X_{\alpha}$, we have

$$
\begin{aligned}
\operatorname{ad}\left(\mathfrak{w}^{-1}(H)\right) \cdot Z & =\left[\mathfrak{w}^{-1}(H), \sum_{\alpha \in \Delta\left(\mathrm{n}^{*}\right)} z_{\alpha} X_{\alpha}\right] \\
& =\sum_{\alpha \in \Delta\left(\mathrm{n}^{*}\right)} \alpha\left(\mathfrak{w}^{-1}(H)\right) z_{\alpha} X_{\alpha}=\sum_{\alpha \in \Delta\left(\mathfrak{n}^{*}\right)}(\mathfrak{m} \alpha)(H) z_{\alpha} X_{\alpha}
\end{aligned}
$$

and, hence,

$$
\operatorname{Exp}\left(\operatorname{ad}\left(\mathrm{m}^{-1}(H)\right)\right) \cdot Z=\sum_{\alpha \in \Delta\left(\mathrm{n}^{*}\right)} e^{(\mathfrak{w} \alpha)(H)} z_{\alpha} X_{\alpha} .
$$

To prove $X=\cup_{\mathfrak{w} \in W^{1}} \mathfrak{w} \bar{N}^{*}$, we need the following fact. See [6].

Fact. Let $Y$ be a compact Kähler manifold which satisfies $H^{1}(Y, \mathbf{C})=$ 0 . Then if a complex connected solvable Lie group $S$ acts holomorphically on $Y$, it always has a fixed point inside any analytic subvariety that $S$ leaves invariant. 
The space $X$ satisfies above assumptions and we can take $T$ as $S$. Then since $\mathfrak{m} \bar{N}^{*}$ is a $T$ invariant Zariski open set, the complement $X^{\prime}=X-\cup_{\mathfrak{w} \in W^{\prime}} \mathfrak{w} \bar{N}^{*}$ becomes a $T$ invariant subvariety. Hence if $X^{\prime}$ is not empty, it must have a $T$ fixed point. But this is a contradiction. This completes the proof.

Since the Lie group $G$ acts on $X=G / P$ from the left side, the space $X$ has many global holomorphic vector fields. For an element $H \in \mathfrak{h}$, let us define a holomorphic vector field $V_{H}$ on $X$ by the rule

$$
\left(V_{H} f\right)(\bar{g})=\lim _{\varepsilon \rightarrow 0} \frac{1}{\varepsilon}\{f(\exp (\varepsilon H) \bar{g})-f(\bar{g})\},
$$

where $\bar{g} \in X$ and $f$ is a local function near $\bar{g}$. Then the above theorem implies that the vector field $V_{H}$ is expressible on $\mathrm{m} \bar{N}^{*}$ in the explicit form

$$
V_{H}=\sum_{\alpha \in \Delta\left(\mathrm{n}^{*}\right)}(\mathfrak{w} \alpha)(H) z_{\alpha} \frac{\partial}{\partial z_{\alpha}}
$$

If $H$ belongs to the Weyl chambers then $0 \neq(\mathfrak{w} \alpha)(H) \in \mathbf{R}$ for all $\mathfrak{w} \in W^{1}, \alpha \in \Delta\left(\mathrm{n}^{*}\right)$. Hence the set of all vanishing points of $V_{H}$ agrees with $W^{1}$ and $V_{H}$ vanishes in the first order there.

Let us quote the following fact from C. Kosniowsky [5].

Fact. Let $M$ be a compact complex manifold of dimension $n$ and $A$ a holomorphic vector field with simple isolated zeros $\left\{\zeta_{1}, \ldots, \zeta_{k}\right\}$. Let us consider the Lie derivative $L_{A}: T_{\zeta}^{*}(M) \rightarrow T_{\zeta}^{*}(M)$ at $\zeta \in\left\{\zeta_{1}, \ldots, \zeta_{k}\right\}$ and denote by $\left\{\theta_{1}(\zeta), \ldots, \theta_{n}(\zeta)\right\}$ its eigenvalues. Then we have

$$
\chi_{p}=\sum_{q}(-1)^{q} h^{p, q}=(-1)^{p} \cdot \#\left\{\zeta_{l} \mid \operatorname{Re} \theta_{j}\left(\zeta_{l}\right)>0\right.
$$

for exactly $p$ indices $j, 1 \leq j \leq n\}$,

where $h^{p, q}=\operatorname{dim} H^{q}\left(X, \Omega^{p}\right)$.

Theorem 2 is well known.

TheOREM 2. Let $X=G / P$. Then the numbers $h^{p, q}$ are determined as follows:

(1) $h^{p, q}=0$ for $p \neq q$,

(2) $h^{p, p}=\left\{\mathfrak{w} \in W^{1} \mid(\mathfrak{w} \alpha)(H)>0\right.$ for exactly $p$ weights $\alpha, \alpha \in$ $\left.\Delta\left(n^{*}\right)\right\}$. 
Proof. (1) has been shown in Lemma 1. By using (4.3) we can easily calculate the eigenvalues of the Lie derivative $L_{V_{H}}$ at the zero point $\mathfrak{w} \in W^{1}$. In fact they are the values $\{2(\mathfrak{m} \alpha)(H)\}_{\alpha \in \Delta\left(n^{*}\right)}$. After noting $\chi_{p}=(-1)^{p} \cdot h^{p, p}$, we complete the proof.

Theorem 3. Let $X=G / P$. Let $\mathcal{E}$ be a homogeneous vector bundle which is induced from a representation $\phi: P \rightarrow G L(V)$. Then:

(1) The vector bundle $\mathcal{E}$ is $V_{H^{-}}$equivariant.

(2) The representative $(-1)^{d} \sigma_{d}\left(\tilde{V}_{H, Z}\right)$ of the dth Chern class, $0 \leq d \leq r$ $=$ rank $\mathcal{G}$, of $\mathcal{E}$ in $H^{0}\left(Z, \Theta_{Z}\right)$ takes the value $\sigma_{d}\left(d \phi\left(\mathfrak{w}^{-1}(H)\right)\right)$ at $\mathfrak{w} \in W^{1}$. Here we denote the differential of $\phi$ by $d \phi: \mathfrak{p} \rightarrow \mathfrak{g l}(V)$.

REMARK. For the line bundle case, i.e., $r=1$, see E. Akyilidiz [1].

Proof. The vector bundle $\mathcal{E}$ is obtained by dividing $G \times V$ by the equivalence relation $(g, v) \sim\left(g p, \phi^{-1}(p) v\right)$ for $g \in G, p \in P, v \in V$. Therefore a local section $v$ of $\varepsilon$ can be interpreted as the $V$-valued function on some open set $U$ of $G$ which satisfies $v(g)=\phi(p) v(g p)$ for $g, g p \in U, p \in P$. Similarly a local function $f$ on $X$ can be considered as the function satisfying $f(g)=f(g p)$. For these $v(g)$ we define

$$
\left(\tilde{V}_{H} v\right)(g)=\lim _{\varepsilon \rightarrow 0} \frac{1}{\varepsilon}\{v(\exp (\varepsilon H) g)-v(g)\} ;
$$

then

$$
\begin{aligned}
& =\lim _{\varepsilon \rightarrow 0} \frac{1}{\varepsilon}\{\phi(p) v(\exp (\varepsilon H) g p)-\phi(p) v(g p)\} \\
& =\phi(p) \lim _{\varepsilon \rightarrow 0} \frac{1}{\varepsilon}\{v(\exp (\varepsilon H) g p)-v(g p)\} \\
& =\phi(p)\left(\tilde{V}_{H} v\right)(g p) .
\end{aligned}
$$

Hence $\left(\tilde{V}_{H} v\right)(g)$ is also a local section of $\mathcal{E}$. On the other hand, let $f$ be a local function; then

$$
\begin{aligned}
\left(\tilde{V}_{H}(f v)\right)(g)= & \lim _{\varepsilon \rightarrow 0} \frac{1}{\varepsilon}\{f(\exp (\varepsilon H) g) v(\exp (\varepsilon H) g)-f(g) v(g)\} \\
= & \lim _{\varepsilon \rightarrow 0} \frac{1}{\varepsilon}\{(f(\exp (\varepsilon H) g)-f(g)) v(\exp (\varepsilon H) g)\} \\
& +\lim _{\varepsilon \rightarrow 0} \frac{1}{\varepsilon}\{f(g)(v(\exp (\varepsilon H) g)-v(g))\} \\
= & \left(V_{H} f\right)(g) v(g)+f(g)\left(\tilde{V}_{H} v\right)(g) .
\end{aligned}
$$


This means $\tilde{V}_{H}$ is a lifting of $V_{H}$ to $\mathcal{E}$. Hence $\mathcal{E}$ is $V_{H}$-equivariant. Let $v(g)$ be a local section of $\mathcal{E}$ which takes a constant vector $v$ along the set $\mathfrak{w} N^{*}$. Then

$$
\begin{aligned}
& \left(\tilde{V}_{H} v\right)(\mathfrak{w} \exp Z)=\lim _{\varepsilon \rightarrow 0} \frac{1}{\varepsilon}\{v(\exp (\varepsilon H) \mathfrak{w} \exp Z)-v(\mathfrak{w} \exp Z)\} \\
& =\lim _{\varepsilon \rightarrow 0} \frac{1}{\varepsilon}\left\{\phi\left(\mathfrak{w}^{-1} \exp (-\varepsilon H) \mathfrak{w}\right)\right. \\
& \qquad \cdot v\left(\mathfrak{w} \mathfrak{w}^{-1} \exp (\varepsilon H) \mathfrak{w} \exp Z \mathfrak{w}^{-1} \exp (-\varepsilon H) \mathfrak{w}\right) \\
& =\lim _{\varepsilon \rightarrow 0} \frac{1}{\varepsilon}\{\phi(\mathfrak{w} \exp Z)\} \\
& =\lim _{\varepsilon \rightarrow 0} \frac{1}{\varepsilon}\left\{\operatorname{Exp}\left(-\varepsilon d \phi\left(\mathfrak{w}^{-1}(H)\right)\right) v-v\right\} \\
& =-d \phi\left(\mathfrak{w}^{-1}(H)\right) v(\mathfrak{w} \exp Z) .
\end{aligned}
$$

Therefore if we choose a basis of local sections of $\mathcal{E}$ on $m \bar{N}^{*}$ from these sections, we can write $\tilde{V}_{H, Z}=-d \phi\left(\mathrm{m}^{-1}(H)\right)$ by using matrix notation. So we have

$$
\begin{aligned}
\operatorname{det}\left(t I-\tilde{V}_{H, Z}\right) & =\operatorname{det}\left(t I-(-d \phi)\left(\mathfrak{w}^{-1}(H)\right)\right) \\
& =\sum_{d=0}^{r}(-1)^{d} \sigma_{d}\left(-d \phi\left(\mathfrak{w}^{-1}(H)\right)\right) t^{r-d} \\
& =\sum_{d=0}^{r} \sigma_{d}\left(d \phi\left(\mathfrak{w}^{-1}(H)\right)\right) t^{r-d} .
\end{aligned}
$$

The proof of Theorem 3 is completed.

\section{REFERENCES}

[1] E. Akyilidiz, Doctor thesis, Univ. of British Columbia.

[2] J. B. Carrell and D. Lieberman, Vector fields and Chern numbers, Math. Ann., 225 (1977), 263-273.

[3] J. B. Carrell and D. Lieberman, Holomorphic vector fields and kähler manifolds, Invent. Math., 21 (1973), 303-309.

[4] J. B. Carrell, Chern classes of the Grassmannians and Schubert calculus, Topology, 17 (1978), 177-182.

[5] C. Kosniowsky, Applications of the holomorphic Lefschetz formulae, Bull. London Math. Soc., 2 (1970), 43-48. 
[6] A. J. Sommese, Holomorphic vector fields on compact kähler manifolds, Math. Ann., 210 (1974), 75-82.

[7] G. Warner, Harmonic Analysis on Semisimple Lie Groups I, II, Springer Verlag.

Received November 6, 1980 and in revised form July 1, 1981.

NAGOYA UNIVERSITY

464 FURO-CHO

CHIKUSAKU

NAGOYA, JAPAN 


\title{
PACIFIC JOURNAL OF MATHEMATICS EDITORS
}

\author{
Donald BabBitT (Managing Editor) \\ University of California \\ Los Angeles, CA 90024 \\ Hugo Rossi \\ University of Utah \\ Salt Lake City, UT 84112 \\ C. C. Moore and Arthur Ogus \\ University of California \\ Berkeley, CA 94720
}

\author{
J. DugundiI \\ Department of Mathematics \\ University of Southern California \\ Los Angeles, CA 90089-1113 \\ R. FINN and H. SAMELSON \\ Stanford University \\ Stanford, CA 94305
}

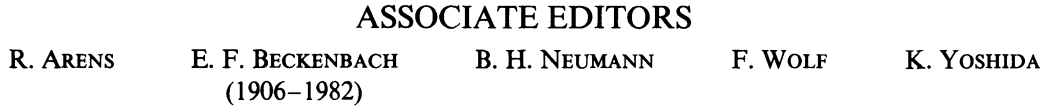

\section{SUPPORTING INSTITUTIONS}

UNIVERSITY OF ARIZONA
UNIVERSITY OF BRITISH COLUMBIA
CALIFORNIA INSTITUTE OF TECHNOLOGY
UNIVERSITY OF CALIFORNIA
MONTANA STATE UNIVERSITY
UNIVERSITY OF NEVADA, RENO
NEW MEXICO STATE UNIVERSITY
OREGON STATE UNIVERSITY

\author{
UNIVERSITY OF OREGON \\ UNIVERSITY OF SOUTHERN CALIFORNIA \\ STANFORD UNIVERSITY \\ UNIVERSITY OF HAWAII \\ UNIVERSITY OF TOKYO \\ UNIVERSITY OF UTAH \\ WASHINGTON STATE UNIVERSITY \\ UNIVERSITY OF WASHINGTON
}

The Supporting Institutions listed above contribute to the cost of publication of this Journal, but they are not owners or publishers and have no responsibility for its content or policies.

Mathematical papers intended for publication in the Pacific Journal of Mathematics should be in typed form or offset-reproduced (not dittoed), double spaced with large margins. Please do not use built up fractions in the text of the manuscript. However, you may use them in the displayed equations. Underline Greek letters in red, German in green, and script in blue. The first paragraph must be capable of being used separately as a synopsis of the entire paper. In particular it should contain no bibliographic references. Please propose a heading for the odd numbered pages of less than 35 characters. Manuscripts, in triplicate, may be sent to any one of the editors. Please classify according to the scheme of Math. Reviews, Index to Vol. 39. Supply name and address of author to whom proofs should be sent. All other communications should be addressed to the managing editor, or Elaine Barth, University of California, Los Angeles, California 90024.

There are page-charges associated with articles appearing in the Pacific Journal of Mathematics. These charges are expected to be paid by the author's University, Government Agency or Company. If the author or authors do not have access to such Institutional support these charges are waived. Single authors will receive 50 free reprints; joint authors will receive a total of 100 free reprints. Additional copies may be obtained at cost in multiples of 50 .

The Pacific Journal of Mathematics is issued monthly as of January 1966. Regular subscription rate: $\$ 132.00$ a year (6 Vol., 12 issues). Special rate: $\$ 66.00$ a year to individual members of supporting institutions.

Subscriptions, orders for numbers issued in the last three calendar years, and changes of address should be sent to Pacific Journal of Mathematics, P.O. Box 969, Carmel Valley, CA 93924, U.S.A. Old back numbers obtainable from Kraus Periodicals Co., Route 100, Millwood, NY 10546.

The Pacific Journal of Mathematics ISSN 0030-8730 is published monthly by the Pacific Journal of Mathematics at P.O. Box 969, Carmel Valley, CA 93924. Application to mail at Second-class postage rates is pending at Carmel Valley, California, and additional mailing offices. Postmaster: Send address changes to Pacific Journal of Mathematics, P. O. Box 969, Carmel Valley, CA 93924.

PUBLISHED BY PACIFIC JOURNAL OF MATHEMATICS, A NON-PROFIT CORPORATION

Copyright $(1983$ by Pacific Journal of Mathematics 


\section{Pacific Journal of Mathematics}

Vol. 108, No. $2 \quad$ April, 1983

Enrique Atencia and Francisco Javier Martin-Reyes, The maximal ergodic Hilbert transform with weights $\ldots \ldots \ldots \ldots \ldots \ldots \ldots \ldots \ldots . \ldots 257$

Bruce Blackadar, The regular representation of local affine motion

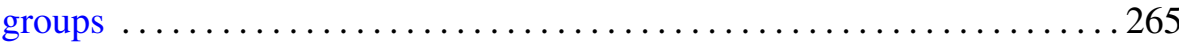

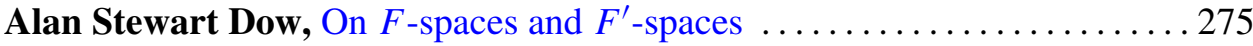

Yoshifumi Kato, On the vector fields on an algebraic homogeneous space . . 285

Dmitry Khavinson, Factorization theorems for different classes of analytic functions in multiply connected domains $\ldots \ldots \ldots \ldots \ldots \ldots \ldots \ldots 295$

Wei-Eihn Kuan, A note on primary powers of a prime ideal . . . . . . . . 319

Benjamin Michael Mann and Edward Yarnell Miller, Characteristic classes for spherical fibrations with fibre-preserving free group

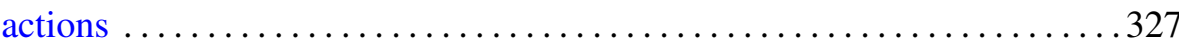

Steven Alan Pax, Appropriate cross-sectionally simple four-cells are flat . . . 379 R. K. Rai, On orthogonal completion of reduced rings ................ 385

V. Sree Hari Rao, On random solutions of Volterra-Fredholm integral

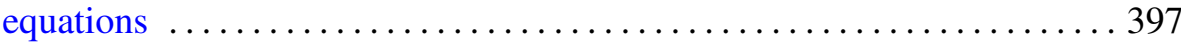

Takeyoshi Satō, Integral comparison theorems for relative Hardy spaces of solutions of the equations $\Delta u=P u$ on a Riemann surface $\ldots \ldots \ldots . .407$

Paul Sydney Selick, A reformulation of the Arf invariant one $\bmod p$ problem and applications to atomic spaces

Roelof Jacobus Stroeker, Reduction of elliptic curves over imaginary quadratic number fields

Jacob Towber, Natural transformations of tensor-products of representation-functors. I. Combinatorial preliminaries

James Chin-Sze Wong and Abdolhamid Riazi, Characterisations of amenable locally compact semigroups 